\title{
Pilot Study of a Device to Induce the Hanger Reflex in Patients with Cervical Dystonia
}

\author{
Takashi AsAHI, ${ }^{1}$ Michi SATO, ${ }^{2}$ Takuto NAKAMURA, ${ }^{2}$ Yuki KON, ${ }^{2}$ \\ Hiroyuki KajIMOTO, ${ }^{2}$ Genko OYAMA, ${ }^{3}$ Akito HAYASHI, ${ }^{4}$ Kazunori TANAKA, ${ }^{5}$ \\ Shunya NAKANE, ${ }^{6}$ Takao TAKESHIMA, ${ }^{7}$ Masami FuJII, ${ }^{8}$ and Satoshi KURODA ${ }^{1}$ \\ ${ }^{1}$ Department of Neurosurgery, Graduate School of Medicine and Pharmaceutical Science, \\ University of Toyama, Toyama, Toyama, Japan; \\ ${ }^{2}$ Department of Informatics, The University of Electro-Communications, Chofu, Tokyo, Japan; \\ ${ }^{3}$ Department of Neurology, Juntendo University, School of Medicine, Tokyo, Japan; \\ ${ }^{4}$ Department of Rehabilitation, Juntendo University Urayasu Hospital, Urayasu, Chiba, Japan; \\ ${ }^{5}$ Department of Neurosurgery, Saiseikai Matsuyama Hospital, Matsuyama, Ehime, Japan; \\ ${ }^{6}$ Department of Neurology, Kumamoto University, School of Medicine, \\ Kumamoto, Kumamoto, Japan; \\ ${ }^{7}$ Department of Neurology, Tominaga Hospital, Osaka, Osaka, Japan; \\ ${ }^{8}$ Department of Neurosurgery, Yamaguchi University, School of Medicine, Ube, \\ Yamaguchi, Japan
}

\begin{abstract}
The hanger reflex (HR) is an involuntary head rotation that occurs in response to a clothes hanger encircling the head and compressing the unilateral fronto-temporal area. Here, we developed an elliptical device to induce the HR and examined its utility for the treatment of cervical dystonia (CD). The study included 19 patients with rotational-type $C D$. The device was applied to each subject's head for at least $30 \mathrm{~min} /$ day for 3 months. Severity scores on part 1 of the Toronto Western Spasmodic Torticollis Rating Scale were evaluated at baseline and after the 3-month trial. Mean scores without and with the device were significantly different both at baseline $(16.6$ vs. 14.7 , respectively; $P<0.05)$ and after the trial $(14.9$ vs. 13.6, respectively; $P<0.05$ ). This preliminary trial suggests that our device can improve abnormal head rotation in patients with $\mathrm{CD}$.
\end{abstract}

Key words: cervical dystonia, hanger reflex, neurorehabilitation

\section{Introduction}

Cervical dystonia (CD) is a type of dystonia in which patients demonstrate an involuntary abnormal head position. The exact cause of CD remains unclear, but it is thought to result from an acquired neurological abnormality. The reported incidence of CD is 1.07 per 100,000 person/years. ${ }^{1)}$

A variety of treatments exist for $\mathrm{CD}$, including electrical stimulation, ${ }^{2)}$ biofeedback, ${ }^{3)}$ physical therapy, ${ }^{4,5)}$ botulinum toxin (BTX) injection, ${ }^{6)}$ spinal cord stimulation, ${ }^{7)}$ deep brain stimulation (DBS), ${ }^{8-10)}$ and selective denervation. ${ }^{11)}$ According to the guidelines of the European Federation of Neurological Societies,

Received: May 15, 2017; Accepted: February 19, 2018

Copyright $@ 2018$ by The Japan Neurosurgical Society This work is licensed under a Creative Commons AttributionNonCommercial-NoDerivatives International License. intramuscular BTX injection is the first-line treatment for $\mathrm{CD} ;{ }^{12)}$ however, BTX treatment requires repeated injections every 3 months and is not financially feasible for some patients. Deep brain stimulation targeting the globus pallidus or subthalamic nucleus is recommended for patients who do not respond to BTX treatment. ${ }^{8-10)}$ While DBS is effective in a proportion of patients, it is both invasive and costly.

When the head is encircled with an ordinary wire clothes hanger (Fig. 1A) and the fronto-temporal region is compressed by the long side of the hanger, reflexive head rotation towards the compressed side occurs (Fig. 1B). ${ }^{13)}$ We named this phenomenon as the hanger reflex (HR). ${ }^{13-15)}$ We induced the HR in patients with $\mathrm{CD}$ and found that it reduced abnormal head rotation, indicating its potential utility for the treatment of CD. ${ }^{16)}$ Accordingly, we developed a portable device for inducing the HR and applied it to a sample of patients with 

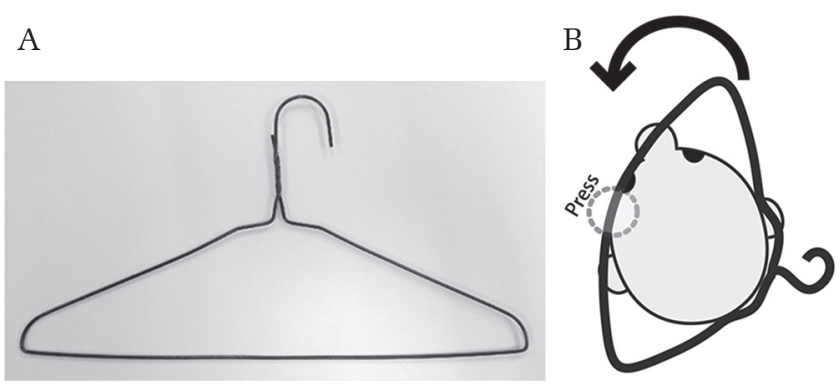

Fig. 1 Schematic of the hanger reflex (HR). (A) Hanger used for this study. An ordinary wire clothes hanger commonly available in Japan was used for HR screening. The hanger was flexible and large enough to encircle the subject's head. (B) Head rotation to the compressed side occurred when the head was encircled with the wire clothes hanger, compressing the fronto-temporal region.

CD (Figs. 2A and 2B). ${ }^{17,18)}$ We then performed a preliminary clinical trial to assess the efficacy of the HR device for the treatment of CD.

\section{Materials and Methods}

\section{Subjects}

A total of 23 patients with rotational-type CD were recruited from 7 facilities in Japan. Written informed consent was obtained from all subjects prior to study participation.

\section{Methods}

During screening, a steel wire hanger was applied to each subject's head to confirm presence of the HR (Figs. 1A and 1B). Our portable HR device was elliptical and made of fiber-reinforced plastic with an interior urethane rim (Fig. 2A). We prepared 7 sizes of the device; the smallest size was $547 \mathrm{~mm}$ in circumference and additional sizes were each $2 \mathrm{~cm}$ larger. A size was selected for each subject based on his or her head circumference. Briefly, the device was applied to the subject's head and rotated in the same direction as the patient's torticollis, to compress the opposite-side fronto-temporal region (Fig. 2B). During the trial, patients self-applied the device for more than 30 min total each day for 3 months. The patients were allowed to complete the 30 min application over multiple periods (e.g., 3 periods of $10 \mathrm{~min}$ each). The patients returned for outpatient follow-up visits each month after starting the trial to confirm proper application of the device and procedural compliance. Scores on the Toronto Western Spasmodic Torticollis Rating Scale (TWSTRS) part 1 (severity scores) were evaluated at baseline and after the 3-month trial period, before and during device application. ${ }^{19)}$ The score was first measured without
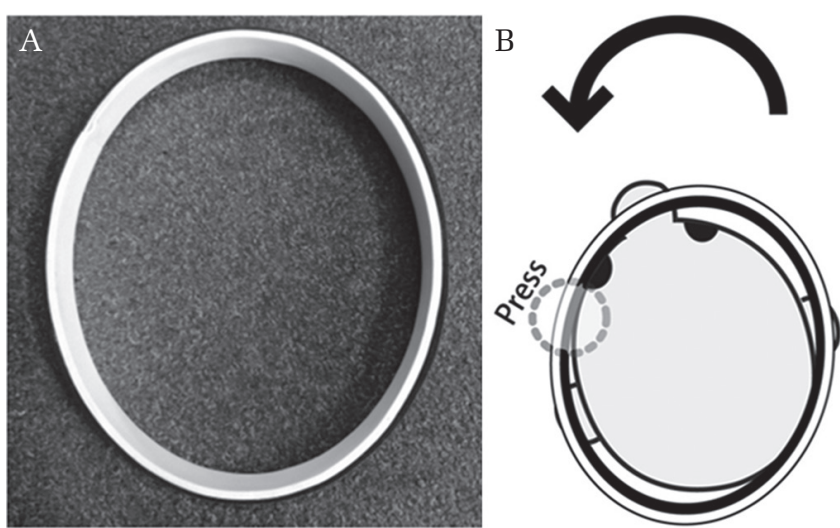

Fig. 2 A portable device for inducing the hanger reflex. (A) The device was elliptically shaped, lightweight, and coated with urethane to prevent skin irritation and to minimize pain and discomfort. (B) Rotation of the device caused compression of the fronto-temporal head region and induction of the hanger reflex. If the patient wears a cap, the device is not visible and is cosmetically acceptable.

the device and subsequently with the device in place. After the conclusion of the trial, a questionnaire was administered to assess any side effects of the device.

\section{Statistical analysis}

All data are expressed as the mean \pm standard deviation. Wilcoxon rank-sum tests were used to compare scores on the TWSTRS part 1 using JMP 11 software (SAS Institute Inc., Cary, NC, USA).

This study was approved by the Ethics Committee of the University of Toyama (ID 23-141) and each institutional ethics committee (trial registration: UMIN 000007772).

\section{Results}

All subjects displayed the HR prior to the trial; however, 4 patients were excluded due to logistical difficulties with attending regular hospital visits. Accordingly, 19 patients (12 men and 7 women) with a mean age of 52.8 years (range, 23-86 years) were included in this study. The median disease duration was 36 months (range, 4-348 months). Figures 3 (line chart) and 4 (boxplots) show TWSTRS part 1 scores before and after the trial, evaluated with and without the device. Mean scores at baseline without and with the device were $16.6 \pm 4.2$ (Fig. 4A) and $14.9 \pm 1.7$ (Fig. 4B), respectively, whereas scores after the 3 -month trial period were $14.7 \pm 4.3$ (Fig. 4C) and $13.6 \pm 4.6$ (Fig. 4D), respectively. The scores were significantly decreased immediately after device application at baseline (Fig. 4, A vs. B [immediate change,] $P<0.05$ ) and at the end of the 3 -month trial period (Fig. 4, C vs. D, $P<0.05$ ). Without application 
25

20

15

10

5
A

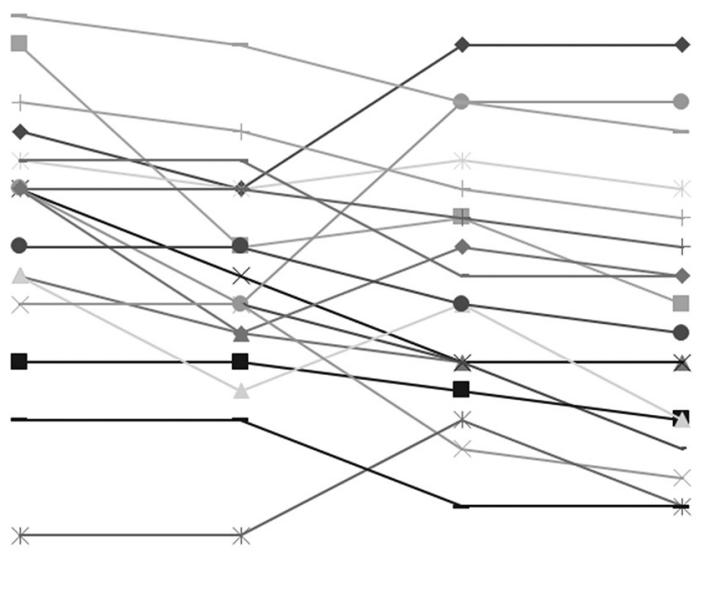

B

C
Fig. 3 Changes in the severity scores of the Toronto Western Spasmodic Torticollis Rating Scale part 1 (line chart). Line chart of baseline scores without (A) and with the device (B), and after the trial without (C) and with the device (D).

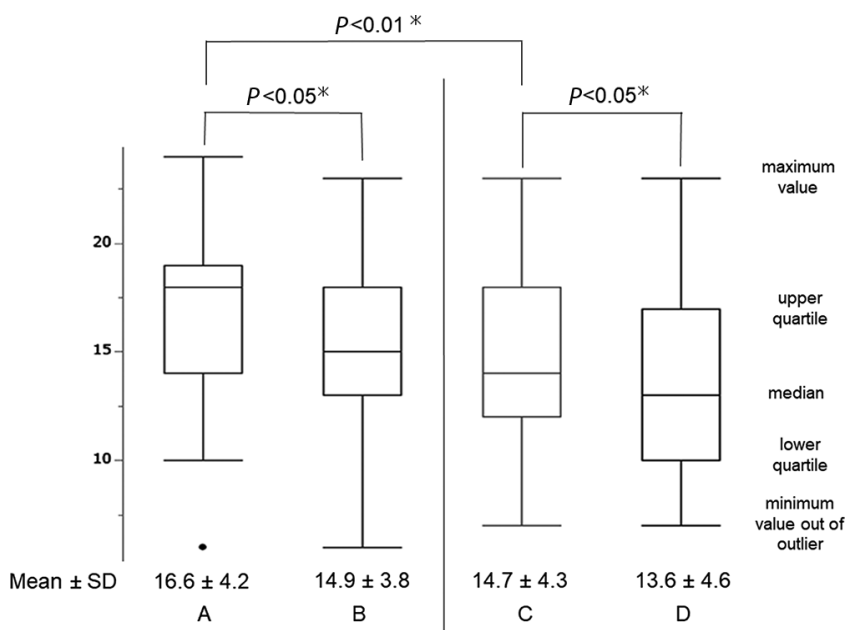

Fig. 4 Changes in the severity scores of the Toronto Western Spasmodic Torticollis Rating Scale part 1 (boxplot). Boxplots of baseline scores without (A) and with the device (B) and after the trial without (C) and with the device (D). (A and B) show the immediate effects of the device, whereas (C and D) demonstrate the absence of a habituation effect over a 3-month period. All changes were statistically significant $(P<0.05)$. Compared to $\mathrm{A}$ and $\mathrm{C}$, the scores were significantly decreased $(P<0.01)$, which means the effect lasted even without using the device (lasting change).

of the device, the scores were significantly decreased between baseline and the end of the 3-month treatment period (Fig. 4, A vs. C [lasting change] $P<0.01$ ). All patients experienced a tolerable level of local pain while wearing the device; but no patient dropped out due to pain. Additionally, there were no skin
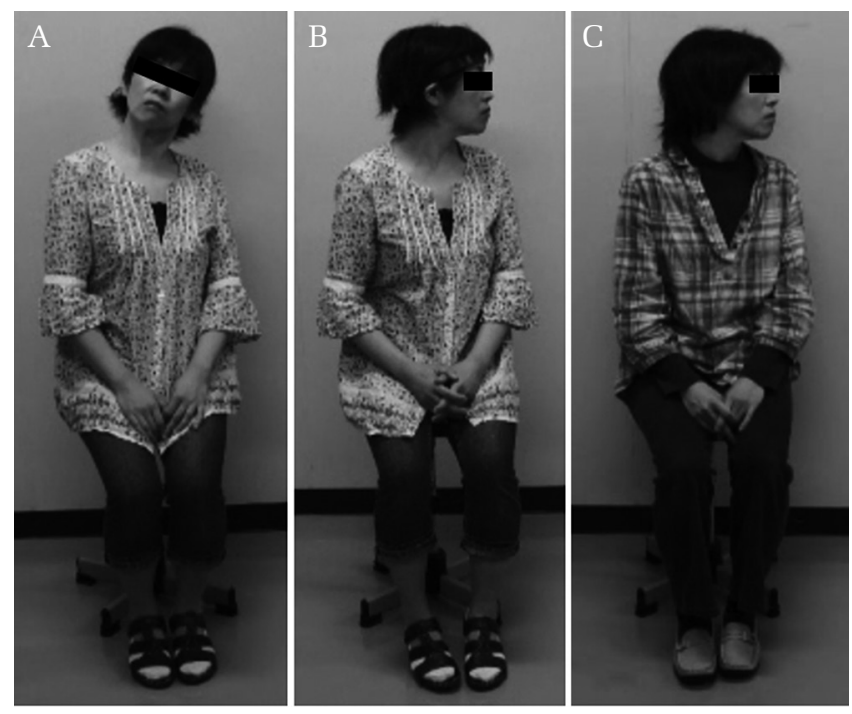

Fig. 5 Illustrative case of cervical dystonia in a 45-yearold woman. (A) At baseline, the patient was unable to turn her head to the left. (B) Immediately after device application, she was able to turn her head to the left (immediate change). (C) After using the device for 3 months, the patient was able to turn her head to the left without use of the device (lasting change).

problems reported in association with device use, such as skin abrasions or other side effects related to device application. None of the participants returned the devices after the trial; rather, they all expressed the desire to continue using the device.

\section{Illustrative case}

A 45-year-old woman presented with a 4-month history of CD. Her head was rotated to the right (patient's right) and she had difficulty rotating to the left (Fig. 5A). The patient's condition was refractory to multiple medications as well as acupuncture. She was then enrolled in the present study. Application of the portable device to the patient's head allowed her to rotate to the left (Fig. 5B). After the 3-month trial, the patient was able to move her head to the left even without application of the device (Fig. 5C). The TWSTRS part 1 scores without and with the device were 23 and 16 at baseline, and 17 and 14 after the 3-month trial period, respectively. The patient did not report any side effects during the trial.

\section{Discussion}

In this study, we observed significant immediate and lasting changes in patients with CD after HR device application. The TWSTRS scores decreased immediately after device application (Fig. 4, A vs. B, immediate change) and after the 3-month trial period, 
even without additional device application (Fig. 4, A vs. C, lasting change). These findings indicate that our HR device not only allowed rotation of the head during use but also improved abnormal head rotation in a long-lasting fashion. The persistent effects of repeated device application on abnormal head rotation (Fig. 4, C vs. D) also indicate that there was no habituation effect to repeated HR induction or to use of our HR device.

The phenomenon named by us as the "hanger reflex" was first reported in 1991. ${ }^{20)}$ Christensen applied a square cardboard box to the heads of 2 patients with spasmodic torticollis at approximately $45^{\circ}$, such that the boxes pressed on the fronto-temporal forehead, and abnormal head rotation was reduced. Matsue et al. demonstrated that this phenomenon was related to non-noxious compression of the fronto-temporal region. ${ }^{14)}$ In 2009, a specialized machine was developed to produce involuntary head rotation by pressing on the fronto-temporal region. ${ }^{15)}$ We previously reported that more than $90 \%$ of healthy volunteers experienced a rotating sensation of the head after fronto-temporal compression, indicating that the HR is common in healthy subjects. ${ }^{13)}$ In the present study, a majority of subjects $(85.4 \%)$ exhibited the HR in the direction of the compressed side; moreover, the HR restricted abnormal head rotation related to CD. In a previous study, the device was shown to generate sufficient pressure on the fronto-temporal region to restrict pathological head rotation even after its use for only 1 month. ${ }^{16)}$

Sensory tricks are well-known phenomena that can temporarily relieve the symptoms of dystonia. We do not believe that the HR is a sensory trick because the HR is present in both normal subjects and patients. Moreover, according to previous studies, the HR requires neither pain nor visual information. ${ }^{14,15)}$ In a previous study, electromyographic (EMG) activity of the sternocleidomastoid muscle (SCM) was monitored in healthy subjects during the HR and showed that when subjects rotated to the left, the application of a hanger to the right fronto-temporal region suppressed EMG activity in the right SCM and caused the subject to assume a neutral position. ${ }^{21)}$ In a patient with CD, the HR suppressed abnormal EMG activity in the inferior obliquus capitis muscle and improved symptoms. ${ }^{21)}$ These findings outline a physiological basis for the HR and suggest that the HR relaxes abnormal muscle contraction in patients with CD.

We believe the underlying mechanism of the HR is a sensory illusion, rather than a sensory trick. We previously hypothesized that the shearing force of a hanger or device applied to the skin of the head induces head rotation. This hypothesis has been supported by work from our co-authors, who developed a spring-loaded lozenge device to generate shearing force on the skin of the head; ${ }^{22)}$ regardless of the region of fronto-temporal compression, the head rotated medially when the skin was sheared to the medial side and laterally when the skin was sheared to the lateral side. With our device, rotation first pulled the skin on the fronto-temporal region to the medial side and then pulled the skin laterally after device release (Fig. 2). We thus suggest that shearing force is required for induction of the HR; specifically, we hypothesize that discomfort related to the shearing force causes subjects to move in the direction of the shearing force to resolve the uncomfortable sensation. Accordingly, when the head is continuously sheared towards the compressed side, the head rotates.

The temporal line demarcates the muscular origin of the temporal muscle, and is located in the frontotemporal region, where it forms an angle on the frontotemporal forehead. In our previous research, the HR was absent in $7.9 \%$ of the 240 trials from 240 volunteers. ${ }^{13)}$ On this basis, non-responders to HR induction may have a more obtuse angle at the temporal line, making it difficult to induce skin shearing force. Our co-authors tentatively named this phenomenon (with unknown mechanism) the "hanger reflex" in 2008. ${ }^{14)}$ After publishing a study on the mechanism of the HR in 2014, ${ }^{22)}$ we believe that the phenomenon is induced by a sensory illusion, and that the HR does not involve a reflex arc of the type that underlies a deep tendon reflex. We discussed whether the term "reflex" should be used, but ultimately decided to continue using the term "reflex" because there were many papers using the term by that time.

While the present study noted promising effects of HR device application in subjects with rotationaltype $\mathrm{CD}$, further studies are necessary to examine the efficacy of this treatment for other types of CD. We found that the HR occurred in the anterior-posterior and lateral directions when the device was applied anterior-posteriorly and laterally, respectively. Similar phenomena have been reported in other regions of the body, including the wrist and waist; ${ }^{2324}$ the HR is thus considered to be a universal bodily phenomenon. Knowledge of this phenomenon has the potential to improve the treatment of neurological disorders, including dystonia in various body parts. The HR device is especially beneficial for patients with CD who cannot afford costly treatments such as BTX or DBS. Our HR device should therefore be considered for use in developing countries with limited financial resources. The modified device has recently become available as a general medical device in Japan (approval number, 35519001). 
There were some limitations to this study. First, only a small number of patients were included in our analysis. Second, given the preliminary nature of this study, and to accommodate the unique demands of patients accustomed to BTX therapy, we used a 3-month observation period. Further improvements might be conferred in a longer trial. Third, the changes in TWSTRS part 1 scores in this study were relatively small; however, these changes were statistically significant over a short observation period. Fourth, this study did not use a control group for comparison, given the difficulty of designing a placebo condition for our experiment. Further research with a large number of patients is required to fully explore the effectiveness and utility of the HR device for the treatment of CD.

\section{Conflicts of Interest Disclosure}

This study was supported by JSPS KAKENHI (23791587) and a Hokugin grant for young scientists. The JSPS KAKENHI and Hokugin grant for young scientists provided unrestricted support and had no role in the oversight or review of the research data or reporting. The authors (TA, KT, MF, and SK) have registered online self-reported COI Disclosure Statement Forms through the website for the Japan Neurosurgical Society members.

\section{References}

1) Steeves TD, Day L, Dykeman J, Jette N, Pringsheim T: The prevalence of primary dystonia: a systematic review and meta-analysis. Mov Disord 27: 1789-1796, 2012

2) Leis AA, Dimitrijevic MR, Delapasse JS, Sharkey PC: Modification of cervical dystonia by selective sensory stimulation. J Neurol Sci 110: 79-89, 1992

3) Jahanshahi M, Sartory G, Marsden CD: EMG biofeedback treatment of torticollis: a controlled outcome study. Biofeedback Self Regul 16: 413-448, 1991

4) Zetterberg L, Halvorsen K, Färnstrand C, Aquilonius SM, Lindmark B: Physiotherapy in cervical dystonia: six experimental single-case studies. Physiother Theory Pract 24: 275-290, 2008

5) Smania N, Corato E, Tinazzi M, Montagnana B, Fiaschi A, Aglioti SM: The effect of two different rehabilitation treatments in cervical dystonia: preliminary results in four patients. Funct Neurol 18: 219-225, 2003

6) Poewe W, Deuschl G, Nebe A, et al.: What is the optimal dose of botulinum toxin $\mathrm{A}$ in the treatment of cervical dystonia? Results of a double blind, placebo controlled, dose ranging study using Dysport. German Dystonia Study Group. J Neurol Neurosurg Psychiatry 64: 13-17, 1998
7) Waltz JM, Scozzari CA, Hunt DP: Spinal cord stimulation in the treatment of spasmodic torticollis. Appl Neurophysiol 48: 324-338, 1985

8) Kiss ZH, Doig-Beyaert K, Eliasziw M, Tsui J, Haffenden A, Suchowersky O; Functional and Stereotactic Section of the Canadian Neurosurgical Society; Canadian Movement Disorders Group: The Canadian multicentre study of deep brain stimulation for cervical dystonia. Brain 130: 2879-2886, 2007

9) Hung SW, Hamani C, Lozano AM, et al.: Long-term outcome of bilateral pallidal deep brain stimulation for primary cervical dystonia. Neurology 68: 457-459, 2007

10) Ostrem JL, Racine CA, Glass GA, et al.: Subthalamic nucleus deep brain stimulation in primary cervical dystonia. Neurology 76: 870-878, 2011

11) Taira T, Hori T: A novel denervation procedure for idiopathic cervical dystonia. Stereotact Funct Neurosurg 80: 92-95, 2003

12) Albanese A, Asmus F, Bhatia KP, et al.: EFNS guidelines on diagnosis and treatment of primary dystonias. Eur J Neurol 18: 5-18, 2011

13) Asahi T, Sato M, Kajimoto H, Koh M, Kashiwazaki D, Kuroda S: Rate of hanger reflex occurrence: unexpected head rotation on fronto-temporal head compression. Neurol Med Chir (Tokyo) 55: 587-591, 2015

14) Matsue R, Sato M, Hashimoto $Y$, Kajimoto H: "Hanger reflex": a reflex motion of a head by temporal pressure for wearable interface. SICE Annual Conference 1463-1467, 2008

15) Sato M, Matsue $R$, Hashimoto $Y$, Kajimoto $H$ : Development of a head rotation interface by using hanger reflex. 18th IEEE International Symposium on Robot and Human Interactive Communication 534-538, 2009

16) Asahi T, Hayashi N, Hamada H, et al.: Application of the hanger reflex to the treatment of cervical dystonia. Funct Neurosurg 49: 173-176, 2010 (Japanese)

17) Kajimoto $H$, Sato $M$, Iguchi $T$, Asahi T: Orthosis. Patent No. WO 2016/052400 A1: 2016 April 7

18) Asahi T, Sato M, Kajimoto H: Orthosis. Patent No. 5552844: 2014 June 6 (Japanese)

19) Comella CL, Stebbins GT, Goetz CG, Chmura TA, Bressman SB, Lang AE: Teaching tape for the motor section of the Toronto Western Spasmodic Torticollis Scale. Mov Disord 12: 570-575, 1997

20) Christensen JE: New treatment of spasmodic torticollis? Lancet 338: 573, 1991

21) Aiba A, Asahi T, Kajimoto $H$, et al.: Botulinum toxin treatment of cervical dystonia with portable needle electromyography and a clinical trial of the hanger reflex. J Mov Disord Disabil 24: 13-18, 2014 (Japanese)

22) Sato M, Nakamura T, Kajimoto H: Movement and pseudo-haptics induced by skin lateral deformation in hanger reflex. The 5th workshop on Telexistence: 2014 (Japanese) 
23) Nakamura $T$, Nishimura $N$, Sato $M$, Kajimoto $H$ : Application of hanger reflex to wrist and waist. 2014 IEEE Virtual Reality (VR), 181-182, 2014

24) Kon Y, Nakamura T, Sato M, Kajimoto H: Effect of hanger reflex on walking. Haptics Symposium 313-318, 2016
Address reprint requests to: Takashi Asahi, MD, PhD, Department of Neurosurgery, Graduate School of Medicine and Pharmaceutical Science, University of Toyama, Sugitani 2630, Toyama, Toyama 930-0194, Japan. e-mail: takashi-tym@umin.ac.jp 